\title{
Actividad antioxidante de Aspidosperma excelsum Benth, Dracontium loretense Krause y Pothemorphe peltata (L) Miq.
}

\section{Antioxidant activity of Aspidosperma excelsum Benth, Dracontium loretense Krause y Pothemorphe peltata (L) Miq.}
Salazar-Díaz, Juan ${ }^{1[\underline{0000-0002-4803-7262]}}$; Guerrero-Marina, Jaime ${ }^{1[0000-0003-}$ 2460-4071] y Rodríguez-Espejo, Yoni ${ }^{10000-0001-5154-1403]}$
${ }^{1}$ Universidad Nacional de San Martín, Tarapoto, Perú jjsalazar@unsm.edu.pe

Resumen. La actividad antioxidante de las tres plantas Remo caspi (Aspidosperma excelsum Benth), Sacha jergón (Dracontium loretense Krause) y Hojas de Santa maría (Pothemorphe peltata (L) Miq.), fue evaluada utilizando el método del DPPH. Se prepararon los extractos hidroalcohólicos mediante maceración exhaustiva durante una semana. Todos los extractos se concentraron bajo vacío hasta su completa sequedad y se guardaron en frascos ámbar, en refrigeración debidamente etiquetada y pesada hasta su siguiente uso. Los resultados de evaluación de la actividad antioxidante de las plantas, la actividad captadora de radicales libres de los extractos se expresó como valor de IC50 (mg/mL) (cantidad necesaria para inhibir la formación de radicales DPPH en un 50\%). El valor bajo de IC50 refleja mejor la acción eliminadora de radicales libres. Aunque la mayoría de las muestras evaluadas mostraron buena capacidad antioxidante con este método (DPPH), los ensayos de los extractos hidro-alcohólicos demuestran que las cortezas de Remo caspi IC50 $(1.84 \mathrm{mg} / \mathrm{mL})$, los cormos de Sacha jergón IC50 $(5.34 \mathrm{mg} / \mathrm{mL})$ y las hojas de Santa maría IC50 $(1.93 \mathrm{mg} / \mathrm{mL})$, según los resultados, se podría considerar como fuentes prometedoras de metabolitos secundarios con actividad antioxidante.

Palabras clave: Actividad antioxidante, Aspinosperma excelsum Benth, Dracontium loretense Krause, poder reductor, Pothemorphe peltata $(L)$ Miq.

Citar como: Salazar-Díaz, J., Guerrero-Marina, J., \& Rodríguez-Espejo, Y. (2021). Actividad antioxidante de Aspidosperma excelsum Benth, Dracontium loretense Krause y Pothemorphe peltata (L) Miq. Revista Agrotecnológica Amazónica, 1(2), 27-39. https://doi.org/10.51252/ra a.v1i2.190

Recibido: $18 / 04 / 2020$

Aceptado: $18 / 06 / 2020$

Publicado: $19 / 07 / 2021$

\begin{abstract}
The antioxidant activity of the three plants Remo caspi (Aspidosperma excelsum Benth), Sacha jergón (Dracontium loretense Krause) and Santa maría leaves (Pothemorphe peltata (L) Miq.), was evaluated using the DPPH method. Hydro-alcoholic extracts were prepared by maceration exhaustive for one week All the extracts were concentrated under vacuum until completely dry and stored in amber bottles, in properly labeled and heavy refrigeration until their next use. The results of the evaluation of the antioxidant activity of the plants the activity of collecting Free radicals of the extracts were expressed as IC50 value $(\mathrm{mg} / \mathrm{mL})$ (amount needed to inhibit DPPH radical formation by 50\%.) The low IC50 value reflects better free radical scavenging action, although most of the evaluated samples showed good antioxidant capacity with this method (DPPH), tests of hydro-alcoholic extracts show that the Ages of Remo Caspi IC50 (1.84mg / mL), Sacha jergón IC50 corms $(5.34 \mathrm{mg} / \mathrm{mL})$ and Santa Maria leaves IC50 (1.93mg / mL), according to the results, it could be considered as promising sources of secondary metabolites with antioxidant activity.
\end{abstract}

Keywords: Aspinosperma excelsum Benth, antioxidant activity, Dracontium loretense Krause, Pothemorphe peltata (L) Miq., reducing power 


\section{$1 \quad$ Introducción}

La lucha contra la oxidación de los alimentos en el curso de su transformación tecnológica, del almacenamiento y distribución, es la problemática que surge la inquietud de conocer cuál es la actividad antioxidante de algunas especies vegetales conocidas comúnmente como Remo caspi, Sacha jergón y Hojas de Santa maría.

Conocedores de que en la mayoría de las especies vegetales se encuentran sustancias como: compuestas polifenólicos, el ácido ascórbico (Vit. C), carotenoides, etc. capaces de atrapar radicales libres evitando la oxidación, se planteó determinar la actividad antioxidante de dichas especies vegetales seleccionadas; teniendo como objetivos la obtención de extractos hidroalcohólicos de las plantas en estudio, determinación de la concentración de fenoles y flavonoides totales, evaluación del poder reductor y actividad antioxidante de los extractos.

Como sustancias antioxidantes destacan: Vitamina E, Vitamina C, Betacarotenos, flavonoides y licopeno, entre otros. Los antioxidantes cumplen funciones protectoras frente a desordenes propios del equilibrio redox del organismo, disminuyendo los efectos adversos que causan radicales libres, principalmente las especies reactivas de oxígeno que se derivan de la respiración celular (Akyol et al., 2016; Bianchi \& Falcioni, 2016).

Para Arosena Chao \& Chavez Cerna (2018) los antioxidantes son compuestos que pueden retardar la oxidación de moléculas, inhibiendo la iniciación y/o propagación de las reacciones en cadena de la formación de radicales libres. Los antioxidantes se dividen en dos categorías principalmente: sintéticos y naturales. Los sintéticos son compuestos de estructuras fenólicas con varios grados de sustitución alquílica, mientras que los naturales pueden ser compuestos fenólicos (tocoferoles, flavonoides y ácidos fenólicos), compuestos nitrogenados (alcaloides, derivados de la clorofila, aminoácidos y aminas) o carotenoides, así como el ácido ascórbico.

En los antecedentes del estudio, Alvarado Chávez (2017) determinó la actividad antioxidante y citotóxica de 35 plantas medicinales de la Cordillera Negra en distintas concentraciones reportando que el IC50 $(\mu \mathrm{g} / \mathrm{mL})$ se pudo realizar a Tecuar, Lengua de perro, Santa lucia y Aliso, por presentar mayor capacidad de captación de radicales libres, demostrando que hay actividad antioxidante en la mayoría de las 35 plantas medicinales investigadas.

Por otra parte, Echavarria et al. (2016) evaluó la capacidad antioxidante de los extractos de 16 plantas medicinales mediante el método DPPH (radical 1,1-difenil-2-picrilhidrazil). La actividad captadora de radicales libres de los extractos se expresó como valor de IC50 $(\mu \mathrm{g} / \mathrm{mL})$ (cantidad necesaria para inhibir la formación de radicales DPPH en un 50\%) aunque la mayoría de las muestras evaluadas mostraron buena capacidad antioxidante con este método (DPPH), los ensayos de los extractos hidro-alcohólicos demostraron que la alcachofa (IC50 9,89 $\mu \mathrm{g} / \mathrm{mL}$ ), 
moringa (IC50 11,4 $\mu \mathrm{g} / \mathrm{mL}$ ) y borraja (IC50 14,0 $\mu \mathrm{g} / \mathrm{mL}$ ) presentaron mayor capacidad antioxidante.

La investigación resulta entonces importante ya que los resultados contribuyen al descubrimiento de nuevas especies vegetales con actividad antioxidante en la provincia y región de San Martín-Perú, lo cual permite avizorar nuevas aplicaciones de la flora amazónica en biocomercio de gran auge en la actualidad, así mismo continuar la búsqueda de otras especies en la exuberante, rica y extensa amazonia peruana.

\section{Materiales y Métodos}

\subsection{Material}

Se utilizaron tres especies vegetales para el estudio: Remo caspi (Aspidosperma excelsum Benth), Sacha jergón (Dracontium loretense Krause) y Hoja de santa maría (Pothemorphe peltata (L) Miq.). Se colectaron aproximadamente $3 \mathrm{~kg}$ de cada especie vegetal de la "Asociación para la conservación y el aprovechamiento sostenible de la biodiversidad" de Chazuta. Estos se secaron en el laboratorio acondicionados para el aire natural, con corriente de aire a la sombra por 15 días, después de procedió a molerlo hasta una malla de 20 mesh.

\subsection{Reactivos}

Se procedió a la preparación de los reactivos para la determinación de la curva patrón, los cuales fueron los siguientes:

Preparación de Solución Stock (ST) de ácido gálico (1g/L): se pesó $25 \mathrm{mg}$ de ácido gálico, y se colocó en una fiola de $25 \mathrm{~mL}$, inmediatamente se aforo con agua destilada.

Preparación de la Solución patrón de ácido gálico $(0,1 \mathrm{mg} / \mathrm{mL})$ : de la solución ST preparada anteriormente se midió $2,5 \mathrm{~mL}$ y diluyo en una fiola de $25 \mathrm{~mL}$ con agua destilada.

Preparación de Solución de Carbonato de sodio al 20\%p: consistió en pesar 5g. de $\mathrm{Na} 2 \mathrm{CO} 3$ y colocar en una fiola de $25 \mathrm{~mL}$ disolviendo con $15 \mathrm{~mL}$ de agua destilada y seguidamente se aforo la fiola.

Preparación de la Solución de Folin Ciocalteu 1 N: consistió en diluir 12,5 mL del reactivo comercial en una fiola $25 \mathrm{~mL}$ aforando con agua destilada y protegiendo de la luz se guardó en frasco color ámbar en refrigeración.

\subsection{Método 1}

Obtención de los extractos hidroalcohólicos: para la extracción por maceración se utilizó alcohol etílico de $70^{\circ} \mathrm{C}$, suficiente cantidad como para cubrir el sólido y aumentar un volumen de $10 \%$ para que este suelto y mejore la extracción. Luego, se filtró los extractos de la misma 
planta, se concentró en rota evaporador, hasta obtener una masa viscosa y para el secado total del extracto se llevó a la estufa a $40^{\circ} \mathrm{C}$. Finalmente, se guardó el sólido seco en un frasco ámbar refrigerada a $4^{\circ} \mathrm{C}$.

Determinación de compuestos fenólicos: se siguió el método de Folin-Ciocalteu, descrito por Gutiérrez Avella et al. (2008) y Doroteo et al. (2013); utilizamos una curva de calibración de ácido gálico con un rango de concentración de 1 a $5 \mathrm{mg} / \mathrm{L}$. Cada uno de los extractos fueron evaluados a una concentración de $0,1 \mathrm{mg} / \mathrm{mL}$. Luego se realizó la lectura de los tubos a $760 \mathrm{~nm}$ y se determinó la recta $\mathrm{Y}=\mathrm{B} \mathrm{X}+\mathrm{C}$ y $\mathrm{R} 2$ = +- 1 (ver Tabla 1 y Figura 1).

\section{Tabla 1}

Cantidad promedio de fenoles totales expresado como ácido gálico equivalente $(A G E)$

\begin{tabular}{|c|c|c|c|c|c|}
\hline \multicolumn{2}{|c|}{ Jergón sacha } & \multicolumn{2}{|c|}{ Santa maría } & \multicolumn{2}{|c|}{ Remo caspi } \\
\hline Abs & $\begin{array}{l}\text { mg AGE/ } \\
\text { g muestra }\end{array}$ & Abs & $\begin{array}{l}\text { mg AGE/ } \\
\text { g muestra }\end{array}$ & Abs & $\begin{array}{l}\text { mg AGE/ } \\
\text { g muestra }\end{array}$ \\
\hline 0,097 & 5,15 & 0,262 & 14,70 & 0,287 & 16,15 \\
\hline 0,059 & 2,95 & 0,575 & 32,82 & 0,311 & 17,53 \\
\hline 0,105 & 5,61 & 0,303 & 17,07 & 0,244 & 13,66 \\
\hline Media & $4,57 \pm 1,42$ & Media & $21,53 \pm 9,98$ & Media & $15,78 \pm 1,96$ \\
\hline $\mathrm{CV}$ & 0,31 & $\mathrm{CV}$ & 0,46 & $\mathrm{CV}$ & 0,12 \\
\hline
\end{tabular}

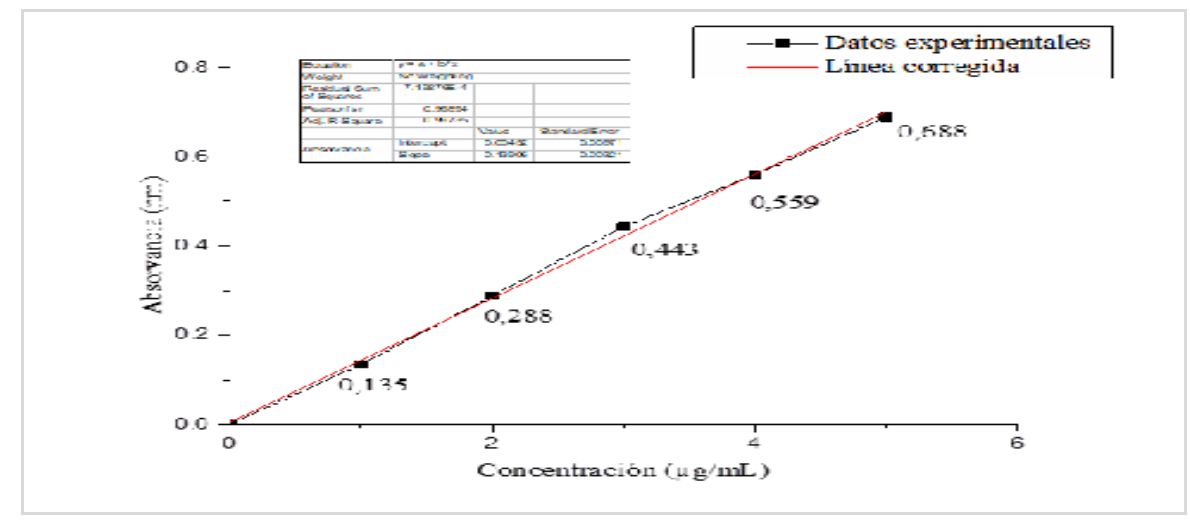

Figura 1: Curva de calibración del Ácido Gálico (AG)

Para determinar los fenoles en las muestras se hizo lo siguiente:

1. $2 \mathrm{mg}$ de extracto seco se disolvió en $50 \mathrm{~mL}$ de agua destilada, y se mezcló adecuadamente verificando que este homogéneo.

2. De la de la disolución preparada, se utilizó $0,50 \mathrm{~mL}$ al cual se agregó $0,75 \mathrm{~mL}$ de Folin Ciocalteu, se dejó en reposo por 5 minutos.

3. Luego se adiciono $0,75 \mathrm{~mL}$ de $\mathrm{Na} 2 \mathrm{CO} 3$ al $20 \%$, agitando adecuadamente hasta homogenizarlo y se dejó en reposo por 90 minutos a temperatura ambiente.

4. Enseguida se hizo las lecturas de absorbancia a $760 \mathrm{~nm}$, se hizo un registro de tres lecturas por cada tubo para corroborar el adecuado funcionamiento del equipo, cuyo resultado en la 
muestra, se expresa en mg de ácido gálico/g de extracto. El mismo procedimiento se realizó con cada extracto de las plantas en estudio

Determinación de Flavonoides: se siguió el método colorimétrico con cloruro férrico, propuesto por Ivanova et al. (2010) también citado por Doroteo et al. (2013); utilizando para la curva de calibración el flavonoide Quercetina, donde se preparan diferentes diluciones de quercetina, se añadió $1,25 \mathrm{~mL}$ de agua destilada seguido de $150 \mu \mathrm{L}$ de nitrito de sodio al $5 \%$, dejando reaccionar por 5 minutos, luego agregamos cloruro de aluminio al 10\%, dejando reposar por 5 minutos, finalmente se añadió $0,5 \mathrm{~mL}$ de $\mathrm{NaOH} 1 \mathrm{M}$ y agua destilada hasta completar un volumen final de reacción de $2,5 \mathrm{~mL}$.

Igual tratamiento se realizó con las muestras y se interpretó la absorbancia en el espectrofotómetro a $510 \mathrm{~nm}$. Se expresó el contenido de flavonoides de las muestras en mg de quercetina/g de extracto hidroalcohólicos (ver Tabla 2 y Figura 2).

Tabla 2

Cantidad promedio de flavonoides totales expresado como quercetina

\begin{tabular}{|c|c|c|c|c|c|}
\hline \multicolumn{2}{|c|}{ Jergón sacha } & \multicolumn{2}{|c|}{ Santa maría } & \multicolumn{2}{|c|}{ Remo caspi } \\
\hline Abs & $\begin{array}{l}\text { mg quercetina/ } \\
\text { g muestra }\end{array}$ & Abs & $\begin{array}{l}\text { mg quercetina/ } \\
\text { g muestra }\end{array}$ & Abs & $\begin{array}{c}\text { mg quercetina/ } \\
\text { g muestra }\end{array}$ \\
\hline 0,017 & 0,52 & 0,043 & 2,02 & 0,038 & 1,74 \\
\hline 0,023 & 0,87 & 0,045 & 2,14 & 0,039 & 1,79 \\
\hline 0,031 & 1,33 & 0,048 & 2,31 & 0,040 & 1,85 \\
\hline Media & $0,90 \pm 0,41$ & Media & $2,16 \pm 0,15$ & Media & $1,79 \pm 0,06$ \\
\hline $\mathrm{CV}$ & 0,449 & $\mathrm{CV}$ & 0,067 & $\mathrm{CV}$ & 0,032 \\
\hline
\end{tabular}

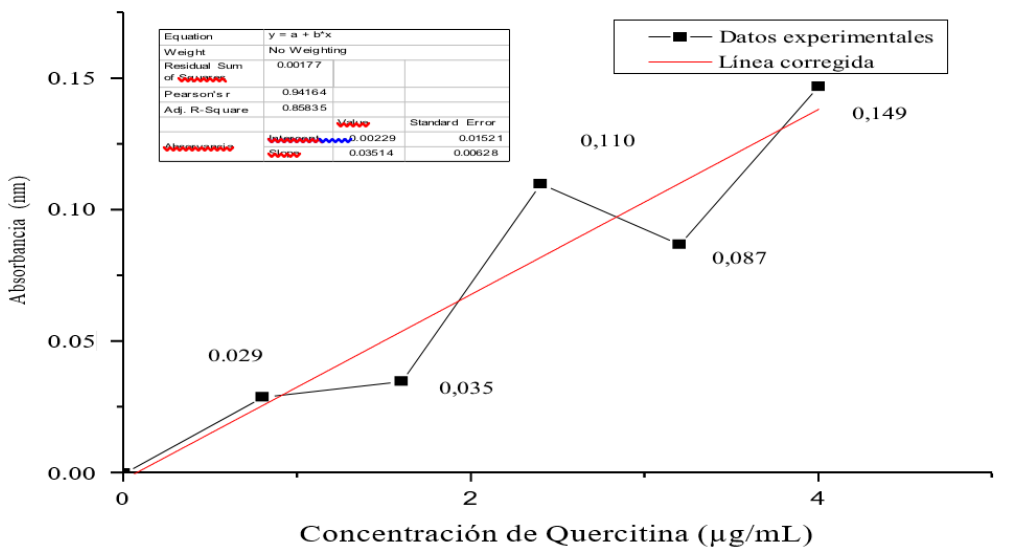

Figura 2: Curva de calibración para flavonoides totales

\subsection{Método 2}

Determinación del poder reductor: esta capacidad de los extractos, de su poder reductor frente a una solución de iones férrico $(\mathrm{Fe} 3+)$ fue medido por el método de Hazra et al. (2008), citado por Doroteo et al. (2013); se dispuso una solución de extractos hidroalcohólicos de concentración de $1 \mathrm{mg} / \mathrm{mL}$ y teniendo una concentración final de 0 a $0,4 \mathrm{mg} / \mathrm{mL}$, fue mezclada 
con $0,5 \mathrm{~mL}$ de solución amortiguadora de $\mathrm{pH}$ 6,6 y $0,5 \mathrm{~mL}$ de solución de hexacianoferrato al $0,1 \%$, incubando a $50^{\circ} \mathrm{C}$ por 20 minutos en baño maría, luego de la reacción, se agregó $0,5 \mathrm{~mL}$ de solución de Tricloroacético (TCA) al 10\%, culminado la primera reacción.

Después $1 \mathrm{ml}$ de la solución anterior final, se agregó $1 \mathrm{~mL}$ de agua destilada y $0,1 \mathrm{~mL}$ de cloruro férrico anhidro al $0,01 \%$ dejando reaccionar por 10 minutos a temperatura ambiente y luego se midió la absorbancia a $700 \mathrm{~nm}$, este proceso se realizó por duplicado con agua destilada como blanco. Una absorbancia superior de la mezcla de reacción indica un poder reductor mayor (ver Tabla 3 y Figura 3).

Tabla 3

Absorbancias de las muestras a diferentes concentraciones en la evaluación del poder reductor

\begin{tabular}{llllll}
\hline & \multicolumn{5}{c}{ Determinaciones } \\
\hline Muestra de Remo Caspi & $\mathbf{1}$ & $\mathbf{2}$ & $\mathbf{3}$ & $\mathbf{4}$ & $\mathbf{5}$ \\
Concentración (mg/mL) & 0,080 & 0,136 & 0,190 & 0,211 & 0,238 \\
Absorbancia & 0,068 & 0,054 & 0,091 & 0,151 & 0,153 \\
& 0,070 & 0,053 & 0,094 & 0,151 & 0,154 \\
& 0,066 & 0,054 & 0,092 & 0,151 & 0,153 \\
Absorbancia Promedio & 0,071 & 0,074 & 0,117 & 0,166 & 0,175 \\
Muestra de Hojas de Santa María & $\mathbf{1}$ & $\mathbf{2}$ & $\mathbf{3}$ & $\mathbf{4}$ & $\mathbf{5}$ \\
Concentración (mg/mL) & 0,080 & 0,136 & 0,190 & 0,211 & 0,238 \\
Absorbancias & 0,025 & 0,080 & 0,139 & 0,166 & 0,167 \\
& 0,028 & 0,083 & 0,145 & 0,167 & 0,167 \\
& 0,023 & 0,076 & 0,129 & 0,166 & 0,167 \\
Absorbancia Promedio & 0,025 & 0,080 & 0,138 & 0,166 & 0,167 \\
Muestra de Jergón sacha & $\mathbf{1}$ & $\mathbf{2}$ & $\mathbf{3}$ & $\mathbf{4}$ & $\mathbf{5}$ \\
Concentración (mg/mL) & 0,080 & 0,136 & 0,190 & 0,211 & 0,238 \\
Absorbancias & 0,071 & 0,078 & 0,095 & 0,080 & 0,111 \\
& 0,071 & 0,078 & 0,094 & 0,081 & 0,111 \\
& 0,071 & 0,079 & 0,096 & 0,083 & 0,112 \\
Absorbancia Promedio & 0,071 & 0,078 & 0,095 & 0,081 & 0,111 \\
\hline
\end{tabular}

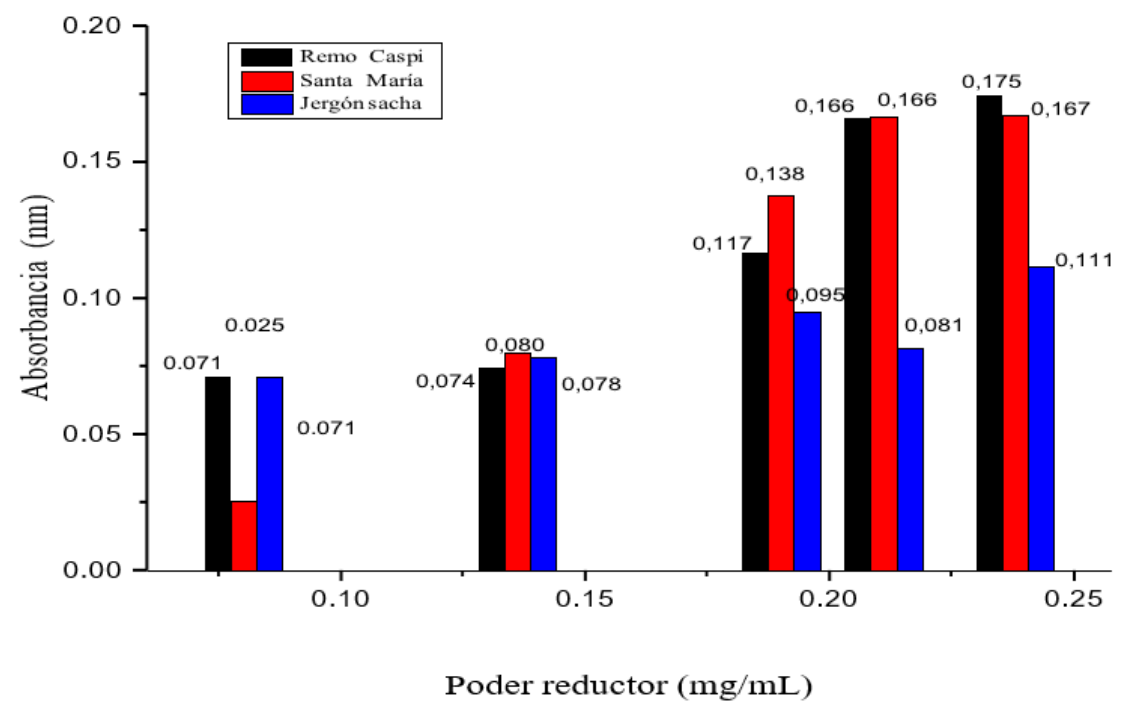

Figura 3: Poder reductor; comparación de las absorbancias de los extractos de Remo caspi (1), San maría (2) y Jergón sacha (3). 
Evaluación de la actividad antioxidante: se realizó mediante la prueba de DPPH conocido como el método de Mensor et al. (2001) también citado por Doroteo et al. (2013), donde se evaluó la actividad antioxidante in vitro de los extractos hidroalcohólicos.

Para lo cual, fue necesario la preparación de diluciones en etanol acuoso de los extractos hidroalcohólicos hasta obtener concentraciones de 0 a 150,0 $\mu \mathrm{g} / \mathrm{mL} .1,0 \mathrm{~mL}$ de cada una de las diluciones con $0,5 \mathrm{~mL}$ de una solución $0,3 \mathrm{mM}$ de DPPH en etanol y se dejó reaccionar a temperatura ambiente por 30 minutos; y luego se procedió a medir la absorbancia de la mezcla a $517 \mathrm{~nm}$., el porcentaje de actividad antioxidante de cada muestra se calculó de acuerdo con la siguiente fórmula:

Actividad antioxidante $(\%)=(\mathrm{AC}-\mathrm{AM}-\mathrm{AB}) / \mathrm{AC}) \times 100$

Donde:

$\mathrm{AM}$ es la absorbancia de la muestra + DPPH, AB es la absorbancia del blanco (muestra + etanol) y AC es la absorbancia del blanco del reactivo (DPPH + etanol).

\subsection{Análisis estadístico}

La investigación fue abordada por un diseño completamente aleatorizado (DCA), al cual fue realizado análisis de la varianza (ANOVA) para estudiar las diferencias entre alas muestras. Para cada prueba, se realizaron tres repeticiones con muestras preparadas utilizando el software estadístico XLSTAT 2009.4.03.

\section{Resultados y discusiones}

Es conocido que la actividad antioxidante puede darse cuanto mayor sea el contenido de polifenoles totales, hay reportes de actividad antioxidante IC50 de $25,12 \mu \mathrm{g} / \mathrm{mL}$ del Dracontium sp (Rivera-Parada, 2013), del Pothemorphe peltata (L) Miq IC50 = 1,2 $\mu \mathrm{g} / \mathrm{mL}$ (Lopes et al., 2013), lo cual corrobora los resultados de fenoles totales con una mayor actividad antioxidante del Pothemorphe peltata (L) Miq; no hay reportes de actividad del Aspidosperma sp , quizás porque la composición fitoquímica de esta especie es mayoritariamente rica en alcaloides indólicos y otros, de actividad mayoritariamente antimalárica según Sosa Amay (2009) y Oliveira et al. (2009).

Bermúdez Riofrío (2017), reporta en su estudio el contenido de flavonoides de la especie Pothemorphe peltata (L) Miq hoy reconocido como Piper peltatum, la cantidad de $564 \mathrm{mg}$ de quercetina/g de extracto seco, también Moyano Aguay (2019) encuentra el contenido de flavonoides en $86,62 \pm 13,67 \mathrm{mg}$ de quercetina/g de extracto estudiando las raíces de la especie de la selva de Ecuador, en ambos casos son cantidades altas respecto a lo encontrado de las hojas de la presente investigación. 
A la concentración de $0,036 \mathrm{~g}$ de extracto $/ \mathrm{ml}$, el poder reductor de todos los extractos tienden a igualar su actividad: un factor notorio del poder reductor de las hojas de santa maría (Pothemorphe peltata (L) Miq.) es mantener un nivel ascendente al aumentar la concentración del extracto, mientras el sacha jergón (Dracontium sp) mantiene un ligero incremento al aumentar la concentración, lo que indicaría que los componentes antioxidantes son mínimos en comparación a la matriz del extracto por tanto, su poder antioxidante será menor; mientras las especies Aspidosperma sp y Pothemorphe peltata (L) Miq. mantienen un nivel de incremento constante de reductor al aumentar la concentración de los extractos, comparando las absorbancias tendrían mayor poder reductor por tanto mayor poder antioxidante.

Como se observa en las tablas 4 y 5, en el ensayo de DPPH de todos los extractos de las distintas plantas el más activo fue el de Sacha Jergón (Dracontium sp) con un valor de IC50 $(5,34 \mathrm{mg} / \mathrm{mL})$ seguido de Santa María (Pothemorphe peltata (L) Miq ) con un IC50 (1,93 mg/mL) y Remo Caspi (Aspidosperma sp) con un IC50 (1,84 mg/mL). La actividad antioxidante es considerable en comparación a los reportes de DPPH IC50 ( $\mu$ g extracto/mL) de Ratania 10,45 \pm 0,48; Uña de gato 12,05 \pm 0,47; Maíz morado 28,89 $\pm 0,81$; Aguaymanto > 100,00; Maca > 100,00; y Yacón 64,52 × 0,66 (Doroteo et al., 2013; Rivera-Parada, 2013).

Tabla 4

Determinación del porcentaje de inhibición del DPPH según concentraciones por cada planta

\begin{tabular}{lrrrrrr}
\hline \multicolumn{1}{c}{$\mathbf{0 , 3 9}$} & $\mathbf{0 , 7 8}$ & $\mathbf{1 , 5 6}$ & $\mathbf{3 , 1 2}$ & $\mathbf{6 , 2 4}$ \\
\hline \multicolumn{1}{c}{ Especie } & $\mathbf{u g} / \mathbf{m l}$ & $\mathbf{u g} / \mathbf{m l}$ & $\mathbf{u g} / \mathbf{m l}$ & $\mathbf{u g} / \mathbf{m l}$ & $\mathbf{u g} / \mathbf{m l}$ \\
\hline $\begin{array}{l}\text { Pothemorphe } \\
\text { peltata } \text { (L) Miq }\end{array}$ & $13 \%$ & $25 \%$ & $43 \%$ & $72 \%$ & $82 \%$ \\
Aspidosperma sp & $14 \%$ & $26 \%$ & $46 \%$ & $73 \%$ & $85 \%$ \\
Dracontium $\mathrm{sp}$ & $6 \%$ & $11 \%$ & $18 \%$ & $31 \%$ & $59 \%$ \\
\hline
\end{tabular}

Tabla 5

Actividad antioxidante de cada planta

\begin{tabular}{lrrrr}
\hline \multicolumn{1}{c}{ Planta } & \multicolumn{1}{c}{ IC50 } & IC50 & IC50 C.A./IC50 & VCEAC 1 g ácido \\
& $\mathbf{m g / 1 0 m L}$ & \multicolumn{1}{c}{$\mathbf{m g / m L}$} & $\begin{array}{c}\text { ácido ascorbico } \\
\text { ascorbico }\end{array}$ \\
\hline Remo Caspi & 0,02 & 1,84 & 17,99 & 17,99 \\
Santa Maria & 0,02 & 1,93 & 21,81 & 21,81 \\
Sacha Jergón & 0,05 & 5,34 & 52,33 & 52,33 \\
\hline
\end{tabular}

Del mismo modo, los resultados se relacionan con el estudio de Echavarria et al. (2016), en el que la actividad captadora de radicales libres de los extractos expresaron como valor de IC50 $(\mu \mathrm{g} / \mathrm{mL})$ buena capacidad antioxidante de los extractos hidro-alcohólicos de la alcachofa (IC50 9,89 $\mu \mathrm{g} / \mathrm{mL}$ ), moringa (IC50 11,4 $\mu \mathrm{g} / \mathrm{mL}$ ) y borraja (IC50 14,0 $\mu \mathrm{g} / \mathrm{mL}$ ).

Santos et al. (2013) hizo una revisión bibliográfica del género Aspidosperma y encontró 20 especies de la selva amazónica, como A. albun, A. Austraje, A. cuspa, A. cylindrocarpon, A. 
vargasii, etc, en la cuales encontró como compuestos quimiotaxonómicos están presentes en todas las especies) a los alcaloides indólicos.

Por otro lado, de Araújo \& de Barros Viana (2020) identificaron en la especie Aspidosperma pyrifolium alcaloides indólicos, compuestos fenólicos (taninos, flavonoides, quinonas); entonces en el extracto etanólico realizado de la planta de estudio Aspidosperma sp. muy probable que contenga alcaloides indólicos y compuestos fenólicos como flavonoides, taninos, quinonas; estos compuestos polares son los que participan en la propiedad de ser antioxidantes.

En base a la búsqueda bibliográfica realizada, se obtuvo un limitado reporte, ya que el análisis de antioxidante para la Aspidosperma sp, es el primer estudio realizado; sin embargo, la investigación realizada por de Araújo \& de Barros Viana (2020) evaluó el poder antioxidante, pero, por otro método, en este caso utilizó una fracción acuosa del extracto etanólico de semillas de A. pyrifolium sobre oxidativo de neutrófilos del metabolismo a través del ensayo de quimioluminiscencia (QL) utilizando luminol (QL lum) y lucigenina (QL luc). El extracto acuoso a concentraciones de 10, 20 y $50 \mu \mathrm{g} / \mathrm{mL}$ fue capaz de reducir la quimioluminiscencia dependiente de luminol, mientras que en la QL dependiente de lucigenina todas las concentraciones reducen la emisión de QL.

Martins et al. (2016) realizaron una investigación de la actividad antioxidante Aspidosperma nitidum, a partir de extractos etanólicos, utilizando un 2,2- ensayo de difenil-1- picrilhidrazilo DPPH, los resultados del IC50 fueron 99,14 $\pm 2,3 \mu \mathrm{g} \mathrm{mL}-1)$ ); y si este valor lo comparamos con el Remo Caspi (Aspidosperma sp) con un IC50 (1,84 $\pm 0,043 \mathrm{mg} / \mathrm{mL})$, vemos que nos da una valor de IC50 menor, entonces se consideraría a la Aspidosperma sp, como una especie con muy buena actividad antioxidante y muy probablemente se deba a los alcaloides y compuestos fenólicos que son los metabolitos quimiotaxonómicos del género Aspidosperma.

Para la especie Pothemorphe peltata se tuvo ausencia de investigaciones como antioxidante, por ello recurimos a buscar información de la familia a la que pertenecen: Ppiperaceae. Asi, encontramos el estudio de Agbor et al. (2005), quienes estudiaron la capacidad antioxidante de 14 hierbas de Camerún. Las muestras liofilizadas extraídas en metanol fueron analizadas usando dos métodos de ensayo de antioxidantes reactivo de Folin-Ciocalteu y el poder antioxidante reductor férrico (FRAP).

Los resultados para las hojas de la especie Piper de la familia Piperaceae, tuvieron actividad antioxidante altos para el método de Folin, así tenemos para la Piper guineense, las hojas tiene un poder oxidante de 491,55 $\pm 9,36$; para la $P$. nigrum, 385,64 $\pm 19,57$. Conparando estos resultados con la especie estudiada Pothemorphe peltata (L) Miq., conocida como Santa María, con un IC50 1,93 $\pm 0,04 \mathrm{mg} / \mathrm{mL}$, vemos que estos valores del poder de antioxidante de la planta estudiada son bajos. 
En cuanto al género Dracontium, la literatura cientifica carece sobre el análisis de antioxidantes, por ello, buscamos en la familia que pertenece: Araceae, y encontramos el estudio de Bello et al. (2020), quienes investigaron especies de la familia Araceae, como la Anchomanes. Difformis, cuya capacidad antioxidante mostró una capacidad más significativa en términos de captación de radicales DPPH (43,64 $\pm 0,94 \mathrm{mg} \mathrm{GAE} / \mathrm{g}$ extracto); y si los comparamos con la especie Dracontium $s p$, resultó $5,34 \pm 0,12 \mathrm{mg} / \mathrm{mL}$. Entonces vemos un poder oxidante muy bajo.

\section{Conclusiones}

Según el poder reductor, se encontró que la concentración de $0,036 \mathrm{~g}$ de extracto/ml, en todos los extractos tienden a igualar su actividad, sin embargo, es notorio que las especies Aspidosperma excelsum Benth y Pothemorphe peltata (L) Miq. tienen una tendencia al incremento de su nivel reductor al aumentar la concentración de los extractos, comparando las absorbancias.

En la evaluación de la actividad antioxidante, se encontró que el mayor porcentaje de inhibición del DPPH fue en Aspidosperma excelsum Benth, seguido de Pothemorphe peltata (L) Miq. Y finalmente el Dracontium loretense Krause corroborado por el valor del IC50 $(\mu \mathrm{g} / \mathrm{mL})$.

\section{Agradecimientos}

Los autores agradecen a la Universidad Nacional de San Martin - Tarapoto, por el aporte financiero para el desarrollo de la presente investigación aprobado con Resolución $\mathrm{N}^{\circ} 138$ 2015-UNSM/CU-R/NLU y por el apoyo con sus laboratorios, equipos y algunos insumos para la ejecución del presente.

Asimismo, a la Asociación de conservación de plantas medicinales y productores artesanales del Distrito de Chazuta, por su participación, proporcionando las plantas medicinales en la cantidad necesaria para la presente investigación.

\section{Referencias bibliográficas}

Agbor, G. A., Oben, J. E., Ngogang, J. Y., Xinxing, C., \& Vinson, J. A. (2005). Antioxidant Capacity of Some Herbs/Spices from Cameroon: A Comparative Study of Two Methods. Journal of Agricultural and Food Chemistry, 53(17), 6819-6824. https://doi.org/10.1021/jf050445c

Akyol, H., Riciputi, Y., Capanoglu, E., Caboni, M. F., \& Verardo, V. (2016). Phenolic Compounds in the Potato and Its Byproducts: An Overview. International Journal of Molecular Sciences, 17(6), 835. https://doi.org/10.3390/IJMS17060835 
Alvarado Chávez, B. (2017). Actividad antioxidante y citotóxica de 35 plantas medicinales de la Cordillera Negra [Universidad Nacional Mayor de San Marcos]. https://hdl.handle.net/20.500.12672/5653

Arosena Chao, M. A., \& Chavez Cerna, R. H. (2018). Evaluación del extracto del fruto de mullak'a (Muehlenbeckia volcanica (Benth.) Endl.) sobre las características de calidad de la carne molida de alpaca (Vicugna pacos) en refrigeración [Universidad Nacional Mayor de San Marcos]. https://hdl.handle.net/20.500.12672/9393

Bello, O. M., Jagaba, S. M., Ogbesejana, A. B., Dada, O. A., Bello, O. E., Kabo, K. S., \& Okunola, J. O. (2020). Antidiabetics, antioxidant, enzyme inhibitory activity and polyphenolic profile of polyphenol rich extracts from three underutilized and indigenous vegetables (UIVs) from Nigeria. Scientific African, 10, e00628. https://doi.org/10.1016/j.sciaf.2020.e00628

Bermúdez Riofrío, J. C. (2017). Evaluación de la actividad antiinflamatoria y citotóxica in vitro de hojas de Piper peltatum L. [Escuela Superior Politécnica de Chimborazo]. http://dspace.espoch.edu.ec/handle/123456789/7938

Bianchi, V. E., \& Falcioni, G. (2016). Reactive oxygen species, health and longevity. AIMS Molecular Science , 3(4), 479-504. https://doi.org/10.3934/MOLSCI.2016.4.479

de Araújo, D. P., \& de Barros Viana, G. S. (2020). Aspidosperma pyrifolium Mart. antioxidants features and neuronal tissues. En Oxidative Stress and Dietary Antioxidants in Neurological Diseases (pp. 189-198). Elsevier. https://doi.org/10.1016/B978-0-12817780-8.00013-X

Doroteo, V. H., Díaz, C., Terry, C., \& Rojas, R. (2013). Compuestos fenólicos y actividad antioxidante in vitro de 6 plantas peruanas. Revista de la Sociedad Química del Perú, 79(1), 13-20. http://www.scielo.org.pe/scielo.php?script=sci_arttext\&pid=S1810634X2013000100003

Echavarria, A., Regnault, H. D., Lisbeth, N., Matute, L., Jaramillo, C., Astudillo, L. R. de, \& Benitez, R. (2016). Evaluación de la capacidad antioxidante y metabolitos secundarios de extractos de dieciséis plantas medicinales. CIENCIA UNEMI, 9(20), 29-35. https://doi.org/10.29076/issn.2528-7737vo19iss20.2016pp29-35p

Gutiérrez Avella, D. M., Ortiz García, C. A., \& Mendoza Cisneros, A. (2008). Medición de Fenoles y Actividad Antioxidante en Malezas Usadas para Alimentación Animal. En Universidad Autónoma de Querétero (Ed.), Simposio de Metrología. https://www.cenam.mx/simposio2008/sm_2008/memorias/m2/sm2008-m220-1108.pdf

Hazra, B., Biswas, S., \& Mandal, N. (2008). Antioxidant and free radical scavenging activity of Spondias pinnata. BMC Complementary and Alternative Medicine, 8(1), 63. https://doi.org/10.1186/1472-6882-8-63

Ivanova, V., Stefova, M., \& Chinnici, F. (2010). Determination of the polyphenol contents in 
Macedonian grapesand wines by standardized spectrophotometric methods. Journal of the Serbian Chemical Society, 75(1), 45-59. https://eprints.ugd.edu.mk/314/

Lopes, A. P., Bagatela, B. S., Rosa, P. C. P., Nanayakkara, D. N. P., Carlos Tavares Carvalho, J., Maistro, E. L., Bastos, J. K., \& Perazzo, F. F. (2013). Antioxidant and Cytotoxic Effects of Crude Extract, Fractions and 4-Nerolidylcathecol from Aerial Parts of Pothomorphe umbellata L. ( Piperaceae ). BioMed Research International, 2013, 1-5. https://doi.org/10.1155/2013/206581

Martins, F. J., Caneschi, C. A., Vieira, J. L. F., Barbosa, W., \& Raposo, N. R. B. (2016). Antioxidant activity and potential photoprotective from amazon native flora extracts. Journal of Photochemistry and Photobiology B: Biology, 161, 34-39. https://doi.org/10.1016/j.jphotobiol.2016.05.012

Mensor, L. L., Menezes, F. S., Leitão, G. G., Reis, A. S., dos Santos, T. C., Coube, C. S., \& Leitão, S. G. (2001). Screening of Brazilian plant extracts for antioxidant activity by the use of DPPH free radical method. Phytotherapy Research, 15(2), 127-130. https://doi.org/10.1002/ptr.687

Moyano Aguay, M. B. (2019). Determinaciòn de la actividad diurética del extracto hidroalcohólico de raíz de Piper peltatum L. en Rattus norvegicus. [Escuela Superior Politécnica de Chimborazo]. http://dspace.espoch.edu.ec/handle/123456789/9706

Oliveira, V. B., Freitas, M. S. M., Mathias, L., Braz-Filho, R., \& Vieira, I. J. C. (2009). Atividade biológica e alcalóides indólicos do gênero Aspidosperma (Apocynaceae): uma revisão. Revista Brasileira de Plantas Medicinais, 11(1), 92-99. https://doi.org/10.1590/S1516-05722009000100015

Rivera-Parada, L. L. (2013). Caracterización fitoquímica, farmacéutica y alimenticia de Papa culebrera india (Dracontium spruceanum (Schott) G.H.Zhu, Araceae) y Sande (Brosimum utile (Kunth) Oken, Moraceae) del Jardín Botánico de Plantas Medicinales del CEA de CORPOAMAZONIA, Mocoa, Putumayo. En Corporación para el Desarrollo Sostenible del Sur de la Amazonia CORPOAMAZONIA (pp. 1-11).

http://www.corpoamazonia.gov.co/files/Investigaciones/Caracterizacion.pdf

Santos, A. C. B., Silva, M. A. P., Santos, M. A. F., \& Leite, T. R. (2013). Levantamento etnobotânico, químico e farmacológico de espécies de Apocynaceae Juss. ocorrentes no Brasil. Revista Brasileira de Plantas Medicinais, 15(3), 442-458. https://doi.org/10.1590/S1516-05722013000300019

Sosa Amay, F. E. (2009). Estudio fitoquímico de la corteza de la raíz de Aspidosperma desmanthum Benth. ex Müll. Arg. con actividad antiplasmodial [Universidad Nacional Mayor de San Marcos]. https://hdl.handle.net/20.500.12672/629 


\section{Conflicto de intereses}

Los autores declaramos que no existen conflictos de interés.

\section{Contribuciones de los autores}

Salazar-Díaz, Juan: Coordinación del proyecto y análisis estadísticos e interpretación.

Guerreo-Marina, Jaime: Toma de muestras en campo y experimentación.

Rodríguez-Espejo, Yoni: Redacción y parte metodológica. 